\title{
PROFILE CHANGES IN THE BINARY PULSAR PSR 1913+16
}

\author{
J. M. WEISBERG \\ Physics and Astronomy Department, Carleton College \\ J. H. TAYLOR \\ Joseph Henry Laboratories and Physics Department, Princeton University
}

\begin{abstract}
According to general relativity, the spin axis of binary pulsar PSR $1913+16$ should precess at a rate of 1.21 degrees per year. This precession will cause the pulse profile to change as our line of sight samples different pulsar latitudes. In order to search for this phenomenon, we have carefully monitored the pulse profile at $1408 \mathrm{MHz}$ for 8.5 years. The ratio of flux density of the first to second pulse component has declined at a rate of approximately $1.65 \%$ per year, with some evidence of a steeper decrease over the past three years. We have detected no evidence for a change in the separation of the two components. We discuss the nature of the pulsar emission region in light of these results.
\end{abstract}

\section{Introduction}

Soon after the discovery of PSR.1913+16, Damour and Ruffini (1974) and Esposito and Harrison (1975) pointed out that the pulsar's spin axis should precess as a result of the metric curvature induced by its companion star. Barker and O'Connell $(1975 a, b)$ were the first to derive an exact expression for the rate of precession of the pulsar's spin axis

$$
\Omega_{\mathrm{p}}=\frac{G^{2 / 3}}{2 c^{2}}\left(\frac{2 \pi}{R_{\mathrm{b}}}\right)^{5 / 3} \frac{m_{\mathrm{c}}}{\left(1-e^{2}\right)} \frac{\left(4 m_{\mathrm{p}}+3 m_{\mathrm{c}}\right)}{\left(m_{\mathrm{p}}+m_{\mathrm{c}}\right)^{4 / 3}}
$$

where $P_{\mathrm{b}}$ is the period of the binary system, $e$ is the orbital eccentricity, and $m_{\mathrm{p}}$ and $m_{\mathrm{c}}$ are the pulsar and companion star masses. For our measured values of these quantities (Taylor and Weisberg 1989), the predicted precession rate is $1.21^{\circ} \mathrm{y}^{-1}$. This precession should lead to observable changes in the pulse profile on a time scale of years.

\section{Observations and analysis}

We have observed the pulse profile of PSR 1913+16 approximately annually since 1981 . The observations were done at the Arecibo Observatory at frequencies near $1400 \mathrm{MHz}$, using identical instrumental configurations whenever possible. We phasealigned and summed all profiles from a given twoweek observing session to create a high signal-tonoise ratio "session grand average" profile for each session. The August 1989 1408-MHz session grand average profile is shown in figure 1.

The two main components of emission from PSR $1913+16$ presumably originate from the edges of a hollow cone of emission centered on the pulsar's magnetic axis (See figure 2). Geodetic precession of

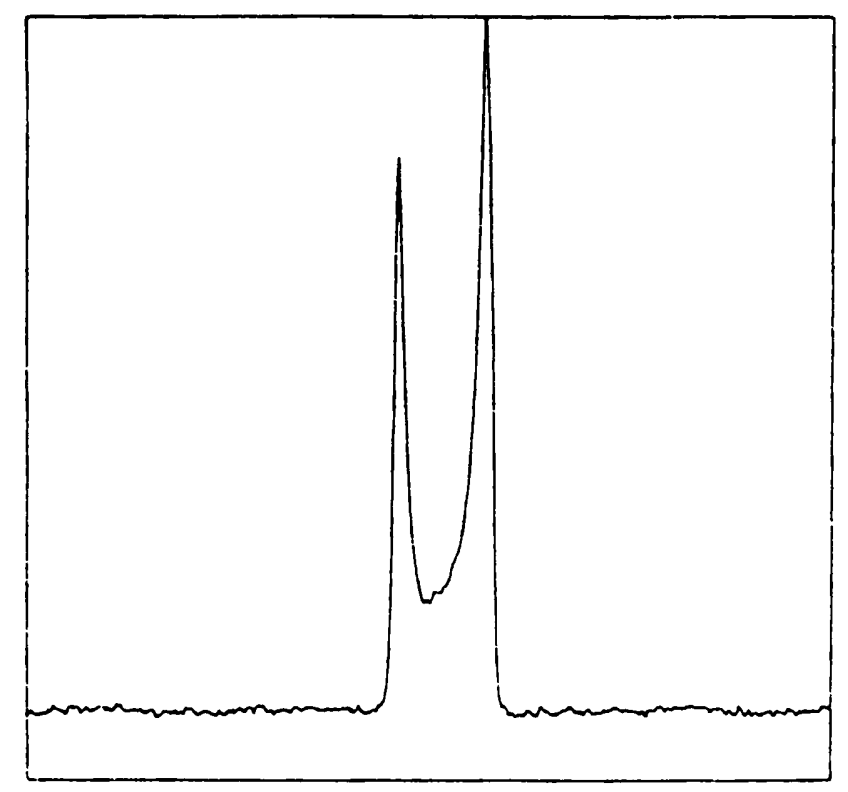

Figure 1 Grand average profile at $1408 \mathrm{MHz}$ for the August 1989 session.

the spin axis should cause a change in the observed profile as our line of sight sarnples different impact parameters during the precession cycle. The most likely expected change is a widening or narrowing of the separation between pulse components as the pulsar's magnetic pole precesses closer to or farther from our line of sight. If the pulsar emission is spatially inhomogeneous, we might also expect to observe variations in the strength or width of the individual components. We have searched for both types of variations, as discussed below.

We fitted Gaussian curves of fixed width to the central portion of each of the two main pulse components. The separation in longitude between the 


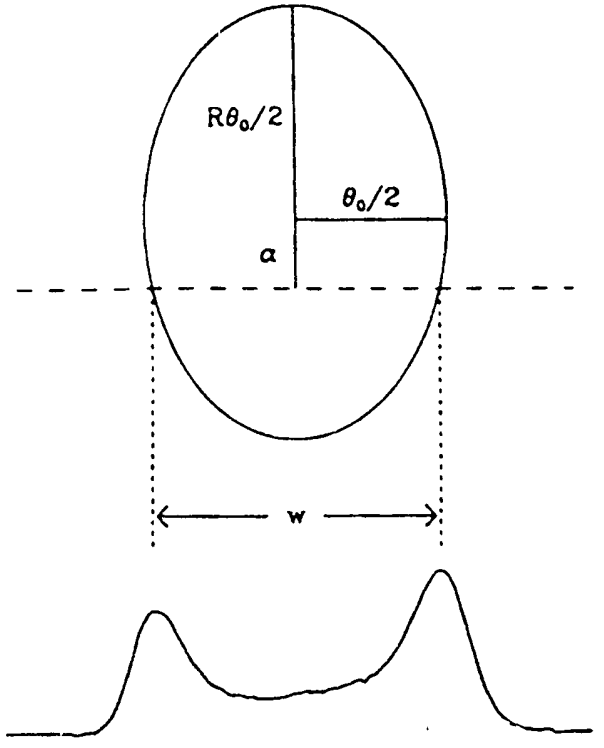

Figure 2 The pulsar's precession carries the line of sight across a hollow elliptical emission cone at impact parameter $\alpha$ from the symmetry axis, as indicated by the horizontal dashed line. The observed pulse profile consists of two components separated by an angle $w$ in longitude.

peaks of the two fitted curves, $w$, is shown in the upper panel of figure 3. There is no evidence for a secular change in the separation between pulse components. The maximum variation in observed component separation in our 8.5 years of observations at $14083 \mathrm{MHz}$ is $|\Delta w|<0.06^{\circ}$.

We now use our upper limit on changes in component separation to constrain the amplitude of precessional motion. We assume an elliptical beam of extent $\theta_{0}$ in longitude and $R \theta_{0}$ in latitude. ${ }^{1}$ The observer's line of sight cuts across this ellipse, making its closest approach at an angle $\alpha$ from the axis of symmetry at the magnetic pole (see figure 2). The two pulse components are separated by a longitude difference $w$. The observed profile, with its two well defined peaks plus some evidence for a central component [especially at lower frequencies (Taylor and Weisberg 1982)], indicates that our line of sight passes very near to the symmetry axis. Hence $\alpha \ll \theta_{0} \approx w$, and $\alpha \approx R\left(w\left(\theta_{0}-w\right) / 2\right)^{1 / 2}$. If indeed $\alpha$ is small, then the component separation $w$ will not vary much with changes in $\alpha$, because the observable parts of the emission zone run nearly perpendicular to the line of sight.

We now establish limits on secular changes in $\alpha$ from our observed upper limit on $\Delta w$. The largest possible change in $\alpha$ for a given $\Delta w$ would be the

\footnotetext{
${ }^{1}$ There is much disagreement on the shape of pulsar beams. Narayan and Vivekanand (1983) found evidence for elongation in latitude $(R>1)$, particularly for shorter-period pulsars like PSR $1913+16$. Other analyses suggest circular conical beams (Lyne and Manchester 1988) or even elongation in the longitude direction (Radhakrishnan and Rankin 1990, Biggs 1990b).
}
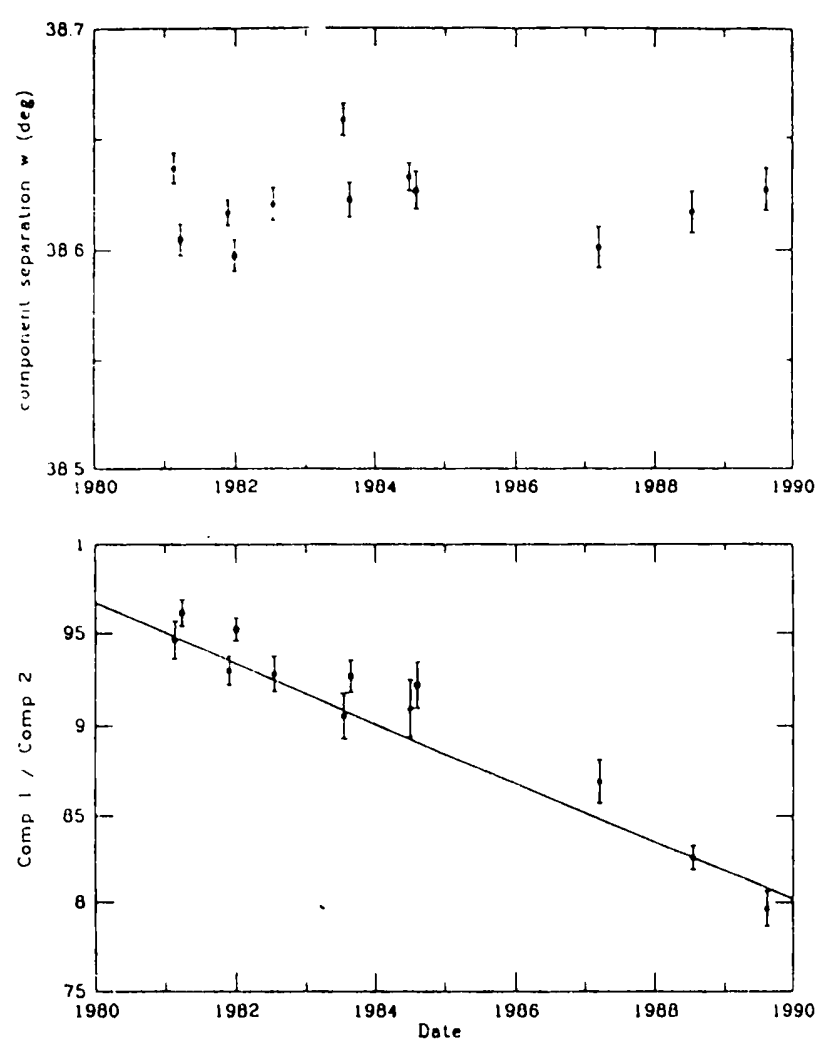

Figure 3 upper panel: Separation between fitted component peaks, and lower panel: flux density ratio of peak 1 to peak 2, plotted as functions of date. Filled squares represent $1408-\mathrm{MHz}$ data; open squares denote $1400-\mathrm{MHz}$ data, arbitrarily shifted by $0.1 \mathrm{y}$ to the right for clarity. The sloping line in the lower plot was fitted to the $1408-\mathrm{MHz}$ data only.

case where the line of sight moves from a small angle on one side of the emission cone axis to a similar angle on the other side. Under these circumstances, the maximum permitted change in $\alpha$ over our $8.5 \mathrm{y}$ observation span is $\Delta \alpha<R(2 w|\Delta w|)^{1 / 2}$. With our measured values of $w=38.6^{\circ}$ and $|\Delta w|<0.06^{\circ}$, $\Delta \alpha<2.2 R^{\circ}$. This gives a limit to the average rate of change $|\dot{\alpha}|=\Delta \alpha / 8.5 \mathrm{y}<0.26 R^{\circ} \mathrm{y}^{-1}$. If $\alpha$ is not near zero, then the limit on $|\dot{\alpha}|$ is substantially smaller.

The rate of change of $\alpha$ will vary throughout the pulsar's precession cycle. It will vanish when the precession carries the pulsar spin axis most directly toward and away from the observer. (See Weisberg, Romani and Taylor 1989 for additional details.) In addition, this quantity may always remain small if the pulsar spin axis is not significantly misaligned with the orbital angular momentum vector. Unfortunately it is not possible at this time to determine the details of the precessional geometry, so we cannot derive more quantitative results. Bailes (1988) did statistical studies of a range of likely progenitor systems. He assumed that the pulsar spin and orbital momenta were aligned before the supernova explosion that formed the second neutron star, and 
that an asymmetric explosion would then impart a kick velocity in a random direction. This kick could then cause a misalignment between the spin and orbital angular momenta. He found a 70 percent likelihood that the value of $|\dot{\alpha}|$ would be less than $0.26^{\circ} \mathrm{y}^{-1}$. Hence our upper limit is still consistent with geodetic precession occurring in the system, but at a rate too small to be detected to date as a change in component separation.

The relative strength of the two main pulse components is changing in a secular fashion, however. As shown in the lower panel of figure 3, the ratio of peak flux density in the two components is decreasing at a rate of one or two percent per year. Weighted least-squares fits to the component ratio data give linear decreases of $1.3 \pm 0.4$ and $1.65 \pm 0.1 \%$ per year at 1400 and $1408 \mathrm{MHz}$, respectively. The $1400-\mathrm{MHz}$ data extend only to 1984 , while $1408-\mathrm{MHz}$ data run through 1989 . The steeper decrease at $1408 \mathrm{MHz}$ is due entirely to the inclusion of post-1984 data, in which the rate of decline is significantly larger. The lower panel of figure 3 displays the line fitted to all the $1408 \mathrm{MHz}$ data. We know of no other pulsar exhibiting long term pulse-shape variations. The most natural explanation for these changes is geodetic precession of the pulsar spin axis.

The observed change in the relative strength of the two pulse components is indicative of the line of sight precessing across rather small patches of emission located along the hollow cone. The individual component widths of $\sim 5^{\circ}$ and the flux decrease rate of $\sim 1.65 \% \mathrm{y}^{-1}$ suggest a change in impact parameter at the rate $|\dot{\alpha}|=0.08^{\circ} \mathrm{y}^{-1}$, which is consistent with the limit obtained above.

\section{Conclusions}

We have found that the pulse profile of PSR $1913+16$ is varying on timescales of a few years. These changes are consistent with geodetic precession of the pulsar spin axis. Although lack of knowledge of the spin axis orientation prevents us from quantifying the precession rate, these observations mark the first detection of this general relativistic phenomenon. While the observed secular variation of the relative strength of the two pulse components could be intrinsic to PSR $1913+16$, we know of no other pulsar exhibiting this behavior. Therefore, we ascribe these changes to the precession across our line of sight of small patches of emission located along the pulsar emission cone.

\footnotetext{
Acknowledgments: We thank Roger Romani for collaborating with us on the earlier stages of this project, and Judith Bartlett, Michael Clarage, Scott Nagle, and Rachel Pildis for assistance with the data analysis. This research was supported by the National Science Foun-
} dation. 